\title{
Pesquisa-ação, pesquisa convergente assistencial e pesquisa cuidado no contexto da enfermagem: semelhanças e peculiaridades
}

\section{Action-research, care-converging research and care-research in nursing context: similarities and peculiarities}

\section{Pesquisa-acción, pesquisa convergente asistencial y pesquisa cuidado en el contexto de la enfermería: Semejanzas y peculiaridades.}

\author{
Maria Ribeiro Lacerda", Clélia Mozara Giacomozzi", Ramone Aparecida Przenyczka"', \\ Tatiana Braga de Camargo'"I
}

\section{RESUMO}

Este estudo tem objetivo de refletir sobre a pesquisa-ação, focada na perspectiva da Enfermagem, buscando suas semelhanças e peculiaridades com a pesquisa-cuidado e a convergente-assistencial. Narra breve histórico dos fundamentos da pesquisa-ação, sinalizando sua origem e seu idealizador, assim como esclarece alguns conceitos e as possibilidades de sua escolha como sustentação metodológica em pesquisas de diversas áreas, especialmente na Enfermagem. Em seguida descrevem-se as características da pesquisa convergenteassistencial e da pesquisa-cuidado, cuja parte dos pressupostos se alicerça na pesquisa-ação, identificando suas interfaces. Ao final, sustentam-se as semelhanças e particularidades com a metodologia da pesquisa-ação, salientando a importância da adoção de uma ou outra, quando o foco de interesse do pesquisador for a transformação de práticas e cuidados, especialmente, quando relacionados a Enfermagem. Enfim, destaca-se a importância da compreensão das diferentes metodologias de pesquisa, uma vez que esse é o primeiro passo para a busca de soluções eficazes para os problemas e inquietações que surgem no desenrolar de uma investigação.

Palavras chave: Enfermagem; Pesquisa; Metodologia; Pesquisa em Enfermagem.

\section{ABSTRACT}

This study aims to reflect upon action-research under a nursing outlook, searching for its similarities and peculiarities with care-research and care-converging research. It entails a brief history of action-research, pointing out its origin and developer, besides clarifying some concepts and possibilities whenever it is chosen as methodological background in research studies from several areas, particularly nursing. Then, the characteristics of care-converging research and care-research whose assumptions partly rely on action-research are put forth, identifying their interfaces. In the end, similarities and peculiarities to care-research methodology are sustained, highlighting the importance to adopt either one whenever the researcher is focused on changes in nursing practice and care. After all, the importance of understanding different research methodologies is pointed out once it is the first step to search for effective solutions to problems and relentlessness while carrying out an investigation.

Key words: Nursing; Research; Methodology; Nursing Research.

\section{RESUMEN}

El objetivo de este estudio es reflexionar sobre la pesquisa-acción, enfocada en la perspectiva de la Enfermería, buscando las semejanzas y peculiaridades con la pesquisa-cuidado y la convergencia-asistencial. Narra una breve historia de los fundamentos de la pesquisaacción, señalando su origen y su idealizador, así como también esclarece algunos conceptos y las posibilidades de su elección como sustentación metodológica en pesquisas de diversas áreas, especialmente en la Enfermería. En seguida son descritas las características de la pesquisa convergencia-asistencial y de la pesquisacuidado, en donde las premisas se basan en la pesquisa-acción, identificando sus conexiones. Al final, se sustentan las semejanzas y

Enfermeira. Doutora em Filosofia de Enfermagem. Coordenadora do Núcleo de Estudos, Pesquisa e Extensão em Cuidado Humano de Enfermagem (NEPECHE) da Universidade Federal do Paraná (UFPR). Professora Adjunta da UFPR. Curitiba/PR. E-mail: lacerda@milenio.com.br.

"Enfermeira. Mestranda no Programa de Pós-graduação em Enfermagem/UFPR.

III Alunas do Curso de Enfermagem da UFPR. Membros do NEPECHE. 
Lacerda MR, Giacomozzi CM, Przenyczka RA, Camargo TB. Pesquisa-ação, pesquisa convergente assistencial e pesquisa cuidado no contexto da enfermagem: semelhanças e peculiaridades. Rev. Eletr. Enf. [Internet]. 2008; 10(3):843-8. Available from: http://www.fen.ufg.br/revista/v10/n3/v10n3a31.htm

particularidades con la metodología de la pesquisa-acción, destacando la importancia de la adopción de una u otra, cuando el foco de interés del investigador es la transformación de prácticas y cuidados, especialmente, cuando están relacionados a la Enfermería. Finalmente, se destaca la importancia de la compresión de las diferentes metodologías de pesquisa, considerando que ese es el primer paso para la búsqueda de soluciones eficaces para los problemas e inquietudes que surgen en el transcurso de una investigación.

Palabras clave: Enfermería; Pesquisa; Metodologia; Pesquisa en Enfermería.

\section{NTRODUÇÃO}

A busca pelo conhecimento é intrínseca à natureza humana. Com o decorrer do tempo, toda a produção oriunda dessa busca originou o saber, desde o comum até o científico, com seus desmembramentos em ciências específicas.

O método científico é adequado aos diferentes tipos de pesquisa, de acordo com o campo, com os sujeitos envolvidos e os objetivos que se pretende alcançar. Assim, as mais diversas áreas têm legitimado suas bases científicas por meio de suas pesquisas. Inclui-se aí a Enfermagem, cujas pesquisas desenvolvidas são cada vez mais freqüentes em muitos países, inclusive no Brasil. Apesar de que no "plano da construção científica, observase a enfermagem com prejuízos indesejáveis pela falta de poder como área de conhecimento face às pressões das políticas de saúde ou pelas instâncias Reguladoras dos órgãos de fomento e amparo à pesquisa"(1). Isto posto, é evidente que a Enfermagem necessita demonstrar cientificamente seu saber, pois se nem as enfermeiras falam do seu conhecimento quem a reconhecerá dotada de conhecimentos científicos? ${ }^{(2)}$

Para tornar seu saber fundamentado cientificamente, a Enfermagem tem se dedicado continuamente a diversas formas de pesquisa. Devido às particularidades dessa profissão, é necessário identificar métodos que sejam compatíveis aos seus fins. A pesquisa de abordagem qualitativa demonstra-se adequada à profissão, visto seu objeto de trabalho: o ser humano. Por meio da pesquisa qualitativa, a profissão obtém ricos detalhes acerca da experiência dos sujeitos em relação a um determinado fenômeno, resultando em descrições densas ou pesadas ${ }^{(3)}$.
Entre tais abordagens de pesquisa mais peculiares aos profissionais enfermeiros, assumem especial importância àquelas onde há interação pesquisador-pesquisado, como a pesquisa participante e a pesquisa-ação, bem como a pesquisa-cuidado e pesquisa convergente-assistencial que possuem traços da pesquisa-ação. Essas diferem dos modelos clássicos quanto ao envolvimento do pesquisador, já que nessa perspectiva este deve adotar uma postura imparcial, colocando-se a parte do objeto pesquisado na tentativa de não interferir, nem interagir com a situação pesquisada ${ }^{(4)}$.

Considerada um processo de pesquisa que estabelece comunicação com a abordagem da pesquisa social, a pesquisa-ação possibilita a troca de informações e a reflexão entre pesquisadores e sujeitos das condições pesquisadas $^{(5)}$. Fato esse, que já torna a pesquisa-ação uma abordagem metodológica específica, o que se afirma sem nuanças de críticas às demais metodologias que também possuem seu valor.

Além disso, a pesquisa-ação é especial porque permite interferências na realidade (e, sempre que possível, alterações que trazem melhorias). Por meio de discussões e elaboração de estratégias, pesquisadores e sujeitos da situação investigada buscam transformações, esclarecimentos e superação dos problemas em um trabalho em conjunto(5).

Considerando a relevância e o modo como as pesquisas se desenvolvem, propõe-se o presente artigo cujo objetivo foi refletir sobre a utilização da pesquisa-ação, na perspectiva da Enfermagem, associando-a e divergindo-a de outros métodos adotados nas pesquisas, especialmente a convergente-assistencial e a pesquisa-cuidado. 
Lacerda MR, Giacomozzi CM, Przenyczka RA, Camargo TB. Pesquisa-ação, pesquisa convergente assistencial e pesquisa cuidado no contexto da enfermagem: semelhanças e peculiaridades. Rev. Eletr. Enf. [Internet]. 2008; 10(3):843-8. Available from: http://www.fen.ufg.br/revista/v10/n3/v10n3a31.htm

\section{FUNDAMENTOS DA PESQUI SA-AÇÃO}

Não há uma uniformidade, entre os diversos autores, quanto à origem da pesquisaação e da indicação precisa de seu ano. Da mesma maneira, não existe um consenso sobre seu idealizador. Às vezes, sua criação é atribuída a Lewin, em 1946, cujas atividades com pesquisa-ação objetivavam as mudanças de hábitos alimentares da população e de atitudes dos americanos frente aos grupos minoritários ${ }^{(6)}$. Mas, destacam-se, também, os empiristas gregos que utilizavam uma fase de pesquisa-ação ${ }^{(7)}$.

Pesquisa-ação é compreendida, na atualidade, como uma forma de pesquisa social com fundamento empírico, concebida e realizada com íntima associação de uma ação ou solução de um problema coletivo, na qual os pesquisadores e os participantes da situação estão envolvidos cooperativamente ${ }^{(5)}$.

Ao invés da busca de informações para mudança em dados quantitativos, essa metodologia trata de encontrar explicações sobre a condição objeto da pesquisa e de vincular pesquisadores e sujeitos envolvidos, além de inserir os pesquisadores no grupo de sujeitos. Assim, não há o predomínio de técnicas ditas convencionais, usadas de acordo com um padrão de observação positivista no qual se manifesta uma preocupação com a quantificação de resultados, com prejuízo da busca pela compreensão e interação entre pesquisadores e membros das situações investigadas $^{(5)}$.

Quando um grupo de sujeitos pede uma transformação aos pesquisadores, a pesquisa é conceituada como pesquisa-ação colaborativa, em que a função do pesquisador será a de fazer parte e cientificizar um processo de mudança anteriormente desatado pelos sujeitos do grupo. Mas, se tal transformação é percebida como indispensável, a partir dos trabalhos iniciais do pesquisador com o grupo e oriunda de um processo que valoriza a construção cognitiva da experiência, tal pesquisa assume um papel de criticidade e, fala-se de pesquisa-ação crítica. Se, no entanto, contrariamente, a transformação é previamente planejada, sem participação dos sujeitos e somente o pesquisador observar os efeitos e avaliar os resultados de sua aplicação, fala-se em pesquisa-ação estratégica ${ }^{(6)}$.

Em outras palavras, a pesquisa-ação crítica não descreve unicamente o que está observando na prática, mas tenta alterar e modificar seu campo de estudo. Para tanto, exige uma cumplicidade entre os sujeitos da pesquisa e os pesquisadores que discutem e refletem sobre as mudanças necessárias; nesse caso, a participação consciente dos sujeitos torna-se relevante. $\mathrm{Na}$ pesquisa-ação colaborativa o problema é identificado pelo grupo que, por sua vez, solicita a intervenção do pesquisador no sentido ajudar a transformar a realidade vivida. Quanto à pesquisa-ação estratégica, o que ocorre é a aplicação de projetos previamente elaborados pelos pesquisadores, sem consideração às mudanças ocorridas no processo da pesquisa.

A partir da metade do século passado, a pesquisa-ação foi utilizada na área educacional com o objetivo de melhorar a prática docente e os resultados educativos. A metodologia pautava-se em uma ação pesquisada, em que se partia da identificação de problemas na escola, buscava-se seus fatores causais, formulava-se uma hipótese de intervenção que era aplicada com os docentes e avaliava-se, coletivamente, as ações empreendidas. Apesar disso, os docentes não eram, na realidade, alçados à condição de pesquisadores, apesar do papel de investigadores ${ }^{(6)}$.

Ponto importante na pesquisa-ação é o planejamento da pesquisa que se dá por meio de três fases. O modelo proposto por Thiollent ${ }^{(5)}$, identifica as seguintes: exploratória; tema da pesquisa; colocação de problemas; o lugar da teoria; hipóteses; seminário; campo de observação, amostragem e representatividade qualitativa; coleta de dados; aprendizagem; saber formal e saber informal; plano de ação; divulgação externa, através das quais organizar-se-á a prática da pesquisa para, dessa forma, obter-se resultados mais confiáveis. Contudo, tal planejamento é maleável, suscetível a alterações, conforme as vivências de um determinado momento.

A Enfermagem, crescente em suas pesquisas, tem utilizado diferentes abordagens metodológicas, inclusive a pesquisa-ação, cuja 
Lacerda MR, Giacomozzi CM, Przenyczka RA, Camargo TB. Pesquisa-ação, pesquisa convergente assistencial e pesquisa cuidado no contexto da enfermagem: semelhanças e peculiaridades. Rev. Eletr. Enf. [Internet]. 2008; 10(3):843-8. Available from: http://www.fen.ufg.br/revista/v10/n3/v10n3a31.htm

influência tem gerado alguns desdobramentos dessa metodologia, como se verá a seguir.

\section{A prática da pesquisa-ação na enfermagem e alguns desdobramentos}

A pesquisa-ação tem sido selecionada para o desenvolvimento de várias pesquisas na Enfermagem $^{(8-9)}$. Com o crescimento destas emergiu o interesse por métodos de pesquisa que compreendessem melhor os sujeitos e os ambientes envolvidos nessa área. Por essa razão, foram desenvolvidos outros métodos baseados nos fundamentos da pesquisa ação, mas apropriados especificamente ao trabalho da Enfermagem, como é o caso da pesquisa convergente-assistencial ${ }^{(10)}$.

A pesquisa convergente-assistencial (PCA) é um tipo de pesquisa que, em seu desenvolvimento, sustenta estreita relação com a situação social e objetiva encontrar soluções para problemas, realizar mudança e introduzir inovações na situação social ${ }^{(10)}$.

A PCA denomina-se desta forma por estar relacionada ao contexto da prática ${ }^{(11)}$. Quando utilizada pela enfermagem, inclui atividades de cuidado/assistência, contudo não se consolida com o ato de cuidar ou de assistir, que é apenas parte do processo de pesquisa. Essa modalidade de pesquisa procura descobrir realidades, resolver problemas ou introduzir inovações num determinado contexto da prática assistencial ${ }^{(10)}$, semelhante à pesquisa-ação que, discute e implementa ações com o fim de melhorar o mundo real.

Obviamente, a PCA segue determinadas fases que nem sempre coincidem com as do modelo proposto pela pesquisa-ação, pois possui como objeto peculiar a prática da Enfermagem. As fases incluem: concepção, instrumentação, perscrutação, análise e interpretação. A diferença fundamental da PCA para a pesquisa ação está ancorada, principalmente, no seu propósito, na forma de escolher o tema e na maneira de conduzir o processo de pesquisa. Na PCA não se estabelece métodos e técnicas específicas de pesquisa, mas se aceita métodos baseados nos mais variados paradigmas da ciência. A observação dos critérios de resolução de problemas introduz inovações no campo da prática, pois esta é desenvolvida concomitantemente com o trabalho do pesquisador, ou este se envolve no trabalho do contexto assistencial da pesquisa. Isso requer envolvimento participativo das pessoas investigadas e reconhece dados obtidos no processo da prática assistencial como dados de pesquisa ${ }^{(10)}$.

Destaca-se, também, a importância da sua fundamentação teórica, pois como a pesquisa-ação, pode sofrer críticas de ser uma metodologia com pouca precisão científica, o que seria uma inverdade, uma vez que, em ambas, deve-se manter rigor em todo seu percurso, inclusive no delineamento do referencial teórico.

Embora seja uma pesquisa no campo assistencial, o que engloba atividades de cuidado/assistência, a PCA deve seguir as exigências de uma investigação científica, com referencial que abrange concepções teóricas, resultados de pesquisas anteriores, estudos de aplicação teórica e artigos de discussão teórica de experiência de prática assistencial ${ }^{(10)}$.

Nesse tipo de pesquisa, o profissional enfatiza 0 pensar e 0 fazer. Primeiro, sistematiza o que faz, diferentemente do profissional que só cuida do cliente e do profissional que visa a desenvolver somente conhecimento científico ${ }^{(10)}$, assim parte da realidade e a ela retorna, no mesmo movimento da pesquisa-ação.

Além da PCA, destaca-se no campo da enfermagem a pesquisa-cuidado $(P C)^{(12)}$. A pesquisa-cuidado surge em decorrência da necessidade de se desenvolver uma metodologia dotada de particularidade à área da Enfermagem, com a qual deve manter uma ligação de reciprocidade e cujos resultados possam subsidiar suas práticas.

O método de PC é fundamentado na dimensão humanista, sendo um instrumento para ajudar o ser-pesquisador e o serpesquisado a um devir harmonioso. A PC sempre se concretiza no cuidado, no conforto do ser-pesquisado, facilita o levantamento de problemas, a observação sistematizada, o diagnóstico de enfermagem, o planejamento e implementação das ações de cuidar e a evolução, num processo intuitivo e científico de cuidar-pesquisar ${ }^{(13)}$. 
Lacerda MR, Giacomozzi CM, Przenyczka RA, Camargo TB. Pesquisa-ação, pesquisa convergente assistencial e pesquisa cuidado no contexto da enfermagem: semelhanças e peculiaridades. Rev. Eletr. Enf. [Internet]. 2008; 10(3):843-8. Available from: http://www.fen.ufg.br/revista/v10/n3/v10n3a31.htm

Em segundo lugar, nos dois métodos está presente a preocupação com o desenvolvimento das relações entre pesquisadores e sujeitos. Por envolver o cuidado de uma pessoa, o pesquisador-cuidador deve agir com muito mais prudência, não só com o sujeito pesquisado, mas com todo o ambiente em que ele se encontra. Contudo, ressalta-se que, na pesquisa-cuidado, o pesquisador é quem decide sobre as ações que serão realizadas, pois é quem detém conhecimento cientifico para tomar as decisões, embora considere a participação do ser pesquisado no seu planejamento.

É preciso que o sujeito pesquisadocuidado seja preparado para o afastamento do ser pesquisador-cuidador. A abordagem teórica na PC tem seu lugar definido em uma fase específica da pesquisa, denominada "estabelecimento das conexões de pesquisa, teoria e prática". Nessa etapa o pesquisador intervém por meio de sua experiência e conhecimentos teóricos. O cuidado ocorre pela junção do conhecimento pessoal e profissional direcionado para a necessidade revelada. Assim, assentam-se a teoria, a prática, a pesquisa e o cuidado. O enfermeiro deve ter características especiais, saber o que fazer e como fazer, ver a situação a partir de uma perspectiva ampla e estar pronto para enfrentar imprevistos frente ao pesquisado-cuidado(13).

Contudo, pela especificidade da PC, temse um método dentro do método, posto que está sujeito às fases do processo de Enfermagem (levantamento de problemas, a observação sistematizada, o diagnóstico de enfermagem, o planejamento e implementação das ações e a avaliação ou evolução).

De todo o exposto, sustenta-se que PCA e PC possuem semelhanças com a metodologia da pesquisa-ação, englobando seus pressupostos essenciais, que são a realização de uma ação que altere uma determinada realidade social, o envolvimento dos pesquisadores e dos sujeitos pesquisados, e a base científica. Claramente, suas fases desenham-se de forma diferente, atendendo os problemas que cada um desses modelos pretende resolver. Embora aplicadas no contexto do trabalho da Enfermagem, são distintas, pois a PC comporta atividades de cuidar e a PCA além de cuidar/assistir, pode englobar qualquer tipo de assistência prestada, além da possibilidade de aplicação em outras áreas de atuação, desde que envolva a prática assistencial.

\section{CONSI DERAÇÕES FI NAIS}

É possível afirmar que a pesquisa-ação é uma metodologia que requer bastante tempo e, apesar de ser foco de algumas críticas, a proposta da pesquisa-ação merece grande apreço, pois valoriza a reflexão e a interação entre pesquisadores e sujeitos da pesquisa. É uma metodologia altamente interativa que intervém na realidade, buscando soluções para os problemas encontrados no campo de pesquisa.

É perceptível que a PCA e a PC apresentam peculiaridades e similaridades frente à pesquisa-ação, até mesmo pelo fato de serem pesquisas advindas da pesquisa-ação. A reflexão acerca de tais aspectos faz-se relevante, visto que para escolha do método de pesquisa é preciso ter claro diferenças e igualdades entre este trio de metodologias.

A PCA, além de não concretizar-se apenas no ato de cuidar/assistir, relaciona-se estreitamente com a situação social, com o intuito de solucionar possíveis problemas, assim ocasionar mudança e introduzir inovações na situação social. Em contrapartida, a PC tem sempre como o foco o cuidado e segue o método do processo de Enfermagem. Para tanto - pesquisador necessita utilizar-se de seus conhecimentos e experiências para uma tomada de decisão acerca do caso do pesquisadocuidado. No que se refere às fases de cada método, tem-se diferenciações entre elas, entretanto, compatível com a necessidade de cada investigação.

Com este estudo, deseja-se esclarecer alguns conceitos que permeiam as classificações da pesquisa, apontando a importância de se utilizar, especialmente, aquelas que possuem maior relação com a Enfermagem. Para que seja possível manter ou não determinadas recomendações sobre as práticas profissionais é importante que os pesquisadores tenham autoridade sobre os métodos de pesquisa, pois sua compreensão é o primeiro passo para a busca de soluções eficazes para os problemas e 
Lacerda MR, Giacomozzi CM, Przenyczka RA, Camargo TB. Pesquisa-ação, pesquisa convergente assistencial e pesquisa cuidado no contexto da enfermagem: semelhanças e peculiaridades. Rev. Eletr. Enf. [Internet]. 2008; 10(3):843-8. Available from: http://www.fen.ufg.br/revista/v10/n3/v10n3a31.htm

inquietações que surgem no desenvolver de uma pesquisa e de uma prática.

\section{REFERÊNCI AS}

1. Carvalho, Vilma de. Acerca da interdisciplinaridade: aspectos epistemológicos e implicações para a enfermagem. Rev. Esc. Enferm. USP 2007;41(3):500-507.

2. Nelson, Sioban. Ciência: o grande segredo da enfermagem. Rev. bras. enferm. 2007; 60(1): 110-111.

3. Driessnack M, Sousa VD, Mendes IAC. An overview of research designs relevant to nursing: part 2: qualitative research designs. Rev. Latino-Am. Enfermagem 2007; 15(4):684688.

4. Gil AC. Métodos e técnicas de pesquisa social. 5a ed. São Paulo: Atlas, 1999.

5. Thiollent M. Metodologia da pesquisa-ação. 13a ed. São Paulo: Cortez, 2004.

6. Franco MAS. Pedagogia da pesquisa-ação. Educação e Pesquisa [Internet]. 2005 [cited 2007 fev 13]; 31(3):483-502. Available from: http://www. scielo.br/pdf/ep/v31n3/a11v31n3.pdf.

7. Tripp D. Pesquisa-ação: uma introdução metodológica. Tradução de Oliveira LL. Educação e Pesquisa [Internet]. 2005 [cited 2007 fev 13]; 31(3):443-66. Available from: http://www. scielo.br/pdf/ep/v31n3/a09v31n3.pdf.

8. Hoga LAK, Reberte LM. Pesquisa-ação como estratégia para desenvolver grupo de gestantes: a percepção dos participantes. Rev. Esc. Enferm. USP. [Internet] 2007 [cited 2008 mar 21]; 41(4). Available from: http://www.scielo.br/pdf/reeusp/v41n4/03.pdf.

9. Silva L, Galera SAF, Moreno V. Encontrandose em casa: uma proposta de atendimento domiciliar para famílias de idosos dependentes. Acta Paul. Enferm., 2007;20(4):397-403.

10. Trentini, Mercedes; Paim, Lygia. Pesquisa em enfermagem. Uma modalidade convergenteassistencial. Florianópolis: Editora da UFSC, 1999. $162 \mathrm{p}$.

11. Oliveira RA, Ciampone MHT. Qualidade de vida de estudantes de enfermagem: a construção de um processo e intervenções. Rev. Esc. Enferm. USP. 2008; 42(1):57-65.

12. Neves EP, Zagonel IPS. Pesquisa-cuidado: abordagem metodológica que integra pesquisa, teoria e prática em enfermagem. In: I
Conferência Internacional do Brasil de pesquisa qualitativa. Taubaté (SP): Centro de Convenções Amacio Mazzaropi, 24-27 de março de 2004. Impresso em CD ROOM, p. 813-820. Produção do Editorial Multimídia Ed. Tec. Art. Realização do Núcleo de Pesquisa da Família Departamento de Psicologia da Universidade de Taubaté.

13. Neves EP, Zagonel IPS. Pesquisa-cuidado: uma abordagem metodológica que integra pesquisa, teoria e prática em Enfermagem. Cogitare Enfermagem [Internet]. 2006 [cited 2007 fev 28]; 11(1):73-9 Available from: http://www.portalbvsenf.eerp.usp.br/pdf/ce/v1 1n1/v11n1a12.pdf.

Artigo recebido em 13.07.07

Aprovado para publicação em 30.09.08 